\title{
Evolution of thermal patterns during steady state evaporation of sessile droplets
}

\author{
Shuang Ye ${ }^{\mathrm{a}}$, Chun-Mei Wu ${ }^{\mathrm{a}}$, Li Zhang ${ }^{\mathrm{a}}$, You-Rong Li ${ }^{\mathrm{a}, *}$, Qiu-Sheng Liu ${ }^{\mathrm{b}}$ \\ a Key Laboratory of Low-grade Energy Utilization Technologies and Systems of Ministry of Education, College of Power Engineering, Chongqing University, Chongqing \\ 400044, China \\ ${ }^{\mathrm{b}}$ Key Laboratory of Microgravity (National Microgravity Laboratory), Institute of Mechanics, Chinese Academy of Sciences, Beijing 100190, China
}

\section{A R T I C L E I N F O}

\section{Keywords:}

Steady state evaporation

Sessile droplet

Thermocapillary flow

Thermal patterns

Infrared thermography

\begin{abstract}
A B S T R A C T
The steady state evaporation process of sessile isopropanol droplets was experimentally investigated by utilizing the infrared thermography. The steady state was achieved by replenishing the droplet from its base. Results indicate that once the evaporation is initiated, the interfacial temperature closed to the triple line is higher than that at the apex, which induces a thermocapillary flow along the interface from the contact line to the apex. With the increase of the evaporation rate, the temperature gradient is enlarged; the flow is enhanced and loses its stability. Then, several thermal patterns, including the forked pattern, the curved hydrothermal waves, the rosebud pattern and the petal-like pattern, were observed. The evolution of thermal pattern is highly correlated with the droplet height and the thermal wave number increases with the substrate temperature. Meanwhile, the global evaporation rate is higher and the surface flow is more intense on copper substrate than that on aluminum substrate.
\end{abstract}

\section{Introduction}

Sessile droplet evaporation is important in cooling [1,2], inkjet printing [3], surface coating [4] and novel medical diagnosis technology [5]. It is also a phenomenon commonly encountered in nature [6]. Moreover, sessile droplet evaporation is a complicated heat and mass transfer process due to the interacted effect among the solid, liquid, gas phases and other boundary phases. Therefore, it is of much practical and full of academic significance to study sessile droplet evaporation.

In the past few decades, sessile droplet evaporation has attracted wide attention in the scientific community. Many researchers devoted themselves to investigating sessile droplet evaporation from various aspects. As early as 1977, Picknett and Bexon [7] had found the possible existence of two evaporation modes throughout the whole life cycle of sessile droplet evaporation, i.e. the constant contact angle (CCA) mode and the constant contact area (CCD) mode. Birdi and Vu [8] studied the evaporation characteristics of water droplets on glass surface. They found that, when the contact angle is less than 90 degrees, the evaporation rate varies linearly with time and the evaporation mode is CCD mode. However, when the contact angle is greater than 90 degrees, the evaporation rate has a nonlinear relationship with time and the evaporation mode is CCA mode. Shanahan and Bourgès
[9] investigated the contact angle characteristics of water droplets on three different polymer surfaces and divided the evaporation process of water droplets on smooth surface into three stages. Hu and Larson [10] studied sessile droplet evaporation with constant contact line by experimental and theoretical calculations. It was shown that the evaporation rate is almost constant when the initial contact angle is less than 40 degrees. Recently, Gleason et al. [11] investigated the steady state evaporation of water droplets placed on a heated substrate and found that the evaporation rate increases with the increase of the contact angle. However, the evaporation mass flux is inverse with the contact angle.

In recent years, the researchers are paying more attention to the coupling effects in the sessile droplet evaporation process, especially liquid flow induced by droplet evaporation. Deegan et al. [6] pointed out that the deposition patterns produced by droplet evaporation are closely related to thermocapillary convection. Hu and Larson [12] confirmed that thermocapillary convection during the evaporation process could restrain the formation of the deposition ring. Ristenpart et al. [13] found that the direction of the surface flow is determined by the relative magnitude of the thermal conductivities of the substrate and the droplet. They also confirmed that thermocapillary convection would affect the deposition pattern. Recently, Sefiane et al. [14] experimentally studied the evaporation process of methanol, ethanol and

\footnotetext{
* Corresponding author.

E-mail address: liyourong@cqu.edu.cn (Y.-R. Li).
} 
FC-72 droplet by utilizing the infrared imager. It was found that the hydrothermal waves appear on the free surface of methanol and ethanol droplets, while the cellular flow pattern arises in the FC-72 droplet. Furthermore, the wave number of hydrothermal waves increases with the liquid volatility and the substrate thermal conductivity. Sobac and Brutin [15] reported that the hydrothermal waves appear in the ethanol evaporation process. Brutin et al. [16] pointed out that there are three stages during the process of droplet evaporation on a heating substrate. Initially, the droplet is in warm-up phase. After the preheating stage, the droplet begins to evaporate with thermal-convective instabilities. At the last stage, the droplet evaporates without any thermal patterns. Sefiane et al. [17] experimentally studied the sessile FC-72 droplet evaporation, and found that the temperature distribution and heat transfer of the substrate will be affected by thermal patterns. Bouchenna et al. [18] investigated the flow pattern inside an evaporating sessile droplet by means of numerical calculation. They found that during the evaporation process, the thermocapillary force is the main driving force of the internal flow pattern. However, at the end of the evaporation process, the strong evaporation around the triple line turns into the main influencing factor of the internal flow pattern. In general, many researchers investigate the liquid flow throughout the whole life cycle of sessile droplet evaporation. However, the droplet shape changing during the evaporation process is not conducive to quantitative analysis of liquid flow.

Up to now, there are only a few reports about the liquid flow in steady state evaporation of sessile droplet. Mahmud and MacDonald [19] observed experimentally the steady state evaporation of sessile water droplet and found that both thermal conduction and thermal convection provide energy for the droplet evaporation. They also found that the convective cell in droplet seems increasing with the substrate temperature. In present work, we reported a series of experiments on the thermal pattern of sessile isopropanol droplet evaporation at steady state. The thermal pattern evolution and the relationship of evaporation rate and liquid flow are exhibited.

\section{Experimental apparatus and procedures}

To observe the thermal patterns at different evaporation conditions, we cautiously designed the experimental apparatus and the experimental procedures to satisfy the need on feeding liquid smoothly, controlling substrate temperature accurately, and measuring droplet surface temperature precisely.

\subsection{Experimental apparatus}

The experimental apparatuses consist of substrate, liquid injection system, temperature control and measurement system, data acquisition system, and droplet shape monitoring system, as shown in Fig. 1(a). The details of substrate are shown in Fig. 1 (b) and (c). It contains two parts: a small substrate and a base substrate. In order to achieve the steady state evaporation, droplets are always entirely covered on the small substrate surface, which is different from the case that droplet evaporating on large flat substrate. The small substrate is glued together with the base substrate. The glue is thermally conductive epoxies (OB101 produced by OMEGA Engineering, INC). The base substrate is made of copper, which can conduct thermal energy to the small substrate rapidly because of the excellent thermal conductivity. Moreover, two different materials, copper and aluminum, are used to make the small substrates. The thermal conductivities of copper and aluminum are different, which are respectively $401 \mathrm{~W} /(\mathrm{m} \cdot \mathrm{K})$ and $237 \mathrm{~W} /(\mathrm{m} \cdot \mathrm{K})$. The radius and the thickness of the small substrate are $R=2.5 \pm 0.05 \mathrm{~mm}$ and $\delta=0.5 \pm 0.05 \mathrm{~mm}$, respectively. The radius of the central hole is $0.25 \pm 0.05 \mathrm{~mm}$. In order to eliminate the effect of surface roughness, both copper and aluminum substrate surfaces were mirror-polished by the company.

The liquid injection system includes a storage bottle and a syringe pump (KDS 200 series). The injection rate of syringe pump ranges from $2.757 \mu \mathrm{l} / \mathrm{h}$ to $70.56 \mathrm{ml} / \mathrm{min}$, which can meet the experimental requirement. The customized storage bottle is made of quartz glass, which is used for storing the pre-prepared isopropanol. The liquid feed tube is passed through the thermostatic bath to keep the feeding liquid and the substrate at the same temperature.

A thermostatic bath (Shanghai Qiqian Electronic Technology Co. Ltd, DC-2006, temperature ranges from $-20^{\circ} \mathrm{C}$ to $100^{\circ} \mathrm{C}$, and the accuracy is $\pm 0.05^{\circ} \mathrm{C}$ ) was used to keep the base substrate temperature constant. Twelve K-type thermocouples with a accuracy of $\pm 0.5^{\circ} \mathrm{C}$ were evenly embedded into the base substrate to monitor the substrate temperature. Their locations are exhibited in Fig. 2. All temperature data from the thermocouples were collected by a multi-channel data acquisition instrument (Agilent, 34972A). During the experiments, the temperature obtained by twelve thermocouples show that the substrate can be heated evenly by the thermostatic bath. In order to measure the droplet surface temperature distribution, an Infrared thermal camera with $25 \mu \mathrm{m}$ lens (FLIR SC325, resolution: $320 \times 240$, the field of view: $8 \times 6 \mathrm{~mm}$, heat sensitivity: $0.05^{\circ} \mathrm{C}$ ) was vertically installed above the substrate. The droplet shape was recorded by the CMOS camera (PixeLINK, PL-B761U, resolution: $752 \times 480$ ). ImageJ (Image Processing and Analysis in Java) was used to measure the droplet height [20]. As the sessile droplet was evaporated in atmospheric environment, a selfdesigned glass cover was installed around the substrate to avoid the disturbance of the ambient air circulation during the experimental process. The experimental fluid is isopropanol, which is supplied by Chongqing Chuandong Co. Ltd. The purity grade of isopropanol is analytical pure grade (Purity greater than 99.7\%). The infrared emissivity of isopropanol liquid is 0.95 [21]. The physical properties at $25^{\circ} \mathrm{C}$ are shown in Table 1.

\subsection{Experimental technique}

Initially, the base substrate temperature was maintained at a constant and uniform value by thermostatic bath. Then, the experimental fluid was carefully prepared and stored in a storage bottle. During the experiment, it was filled into to the syringe without exposing it to air. After that, the fluid was continuously pumped by syringe pump to form a droplet on the small substrate. At the beginning of the droplet evaporation, we adjusted the injection rate of syringe pump based on the real-time monitoring droplet shape according to the CMOS camera. It was considered to be steady-state evaporation when the variation of the droplet height measured by Image $J$ is no more than $45 \mu \mathrm{m}$ for half an hour. Then, the Infrared thermal camera was used to measure the temperature field of the droplet surface. It should be pointed out that the isopropanol is semi-transparent to infrared at the wavelengths of (7-14) $\mu \mathrm{m}$, which means that the temperature distribution captured by the infrared camera is the temperature signature of the liquid layer very close to the droplet surface. Moreover, it is worth mentioning that the infrared thermal imaging technology has been applied in many sessile droplet evaporation researches [22-24]. Therefore, it is reliable to capture the surface temperature in present experiments.

\section{Results and discussion}

\subsection{Thermal patterns induced by droplet evaporation}

The experiments were carried out at atmospheric environment. During the experiment, we always set the ambient temperature at $26 \pm 1{ }^{\circ} \mathrm{C}$ and only adjust the substrate temperature to change the temperature difference between substrate and ambient.

As shown in Fig. 3, the temperature closed to the triple line is always higher than that at the apex via the analysis of the surface temperature distribution captured by the infrared thermal imager. Fig. 3(a) shows the infrared image of droplet surface, in which the red dotted circle represents the edge of the droplet. The temperature distribution 


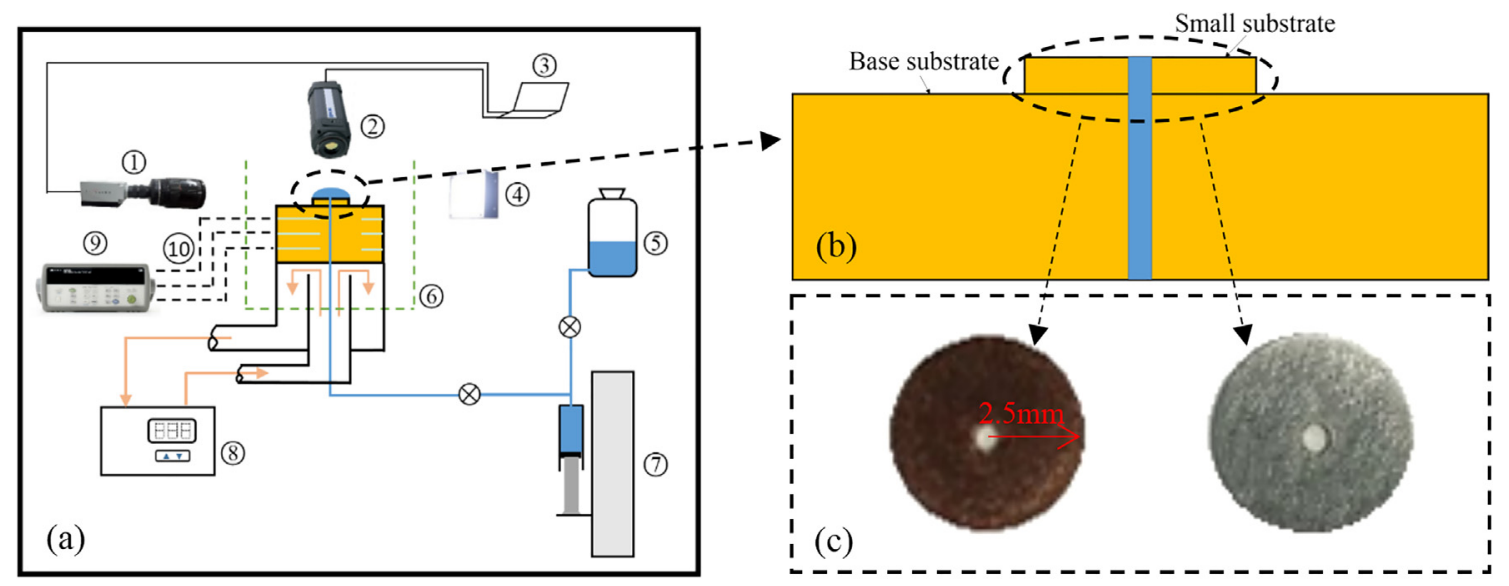

Fig. 1. (a) Schematic of the experimental apparatus. (b) Details of two substrates. (c) Details of small substrate. (1) CMOS camera, (2) Infrared thermal imaging system, (3) Computer, (4) Light source, (5) Liquid storage bottle, (6) Glass cover, (3) High precision syringe pump, (8) Thermostatic bath, (9) Data acquisition system, (10) K-type thermocouple.
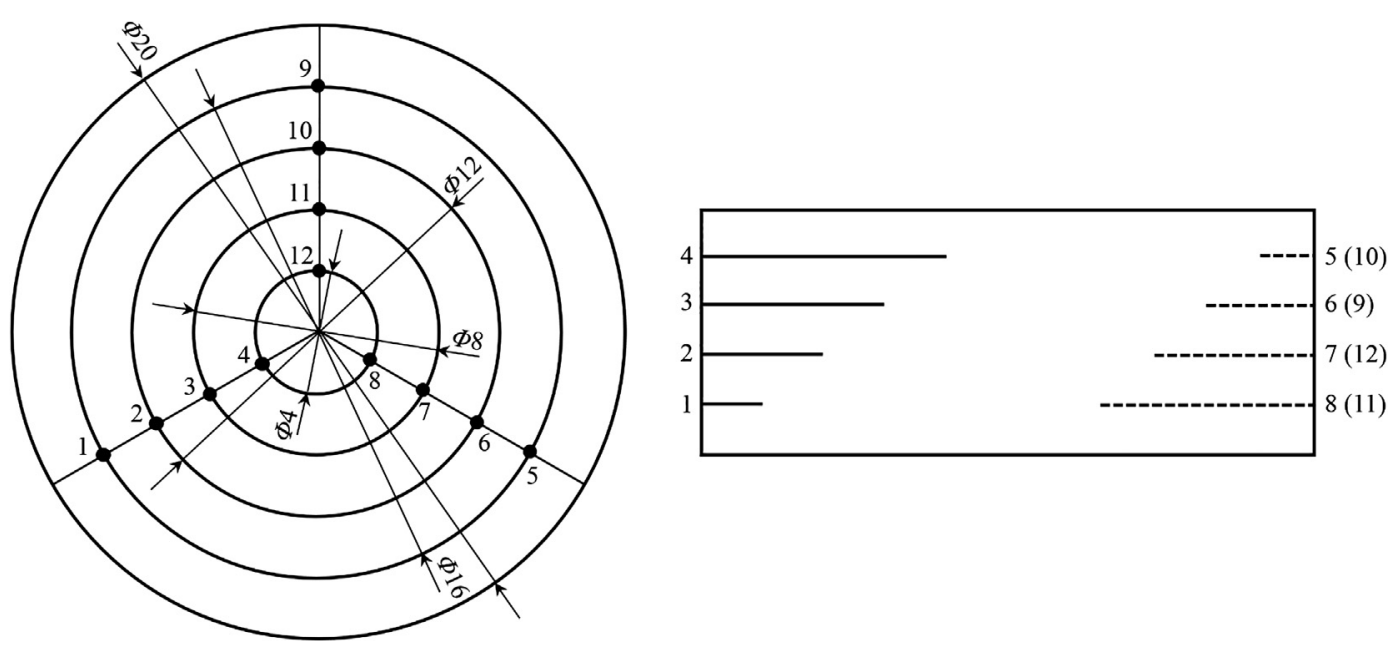

Fig. 2. The locations of twelve thermocouples.

Table 1

Physical properties of isopropanol at $25^{\circ} \mathrm{C}$.

\begin{tabular}{lll}
\hline Parameter & Value & Unit \\
\hline Boiling point & 82.5 & ${ }^{\circ} \mathrm{C}$ \\
Melting point & -88.5 & ${ }^{\circ} \mathrm{C}$ \\
Relative density & 0.79 & $\mathrm{~g} / \mathrm{ml}$ \\
Dynamic viscosity & 1.96 & $\mathrm{mPa} \cdot \mathrm{s}$ \\
Vaporization heat & 745.1 & $\mathrm{~kJ} / \mathrm{kg}$ \\
Specific heat & 2.57 & $\mathrm{~kJ} / \mathrm{kg} \cdot \mathrm{K})$ \\
Thermal conductivity & 0.17 & $\mathrm{~W} /(\mathrm{m} \cdot \mathrm{K})$ \\
\hline
\end{tabular}

along the line 1 in Fig. 3(b) is obtained from Fig. 3(a). Obviously, the lowest temperature appears near the droplet apex. It indicates that there is a temperature gradient along the free surface of the droplet. The value of surface tension is a function of temperature, which can be given for isopropanol as follows [25]

$\gamma=22.9-0.0789 T$

where $\gamma$ is the surface tension in dyn $\cdot \mathrm{cm}^{-1}$, and $T$ is the temperature in ${ }^{\circ} \mathrm{C}$. It is worth mentioning that Eq. (1) is an empirical formula. In this case, the surface tension near the triple line is lower than that near the apex. Thus, the thermocapillary force caused by the surface tension gradient drives the liquid flow from the contact line to the apex. Accordingly, if the temperature distribution on the droplet surface is uniform, there is no surface flow.
The substrate and the sessile droplet shape captured by CMOS camera are presented in Fig. 4. In addition, the droplet height $h$ is defined as the distance between the small substrate surface and the droplet apex. When the substrate temperature $\left(T_{\mathrm{w}}\right)$ is fixed at $40{ }^{\circ} \mathrm{C}$, the thermal patterns on the droplet surface are in variation with the droplet height $h$, as shown in Fig. 5. In this case, three kinds of the thermal patterns appear orderly with the decrease of the droplet height. As exhibited in Fig. 5(a), when the droplet height is $h=1 \mathrm{~mm}$, there is almost no temperature fluctuation on the surface. Actually, when the droplet height is more than $1 \mathrm{~mm}$, there is no distinct temperature fluctuation as well. The thermal conductive path increases with the droplet height. It may cause a larger temperature difference between the substrate and the droplet apex. In this case, the surface flow may be enhanced. Therefore, the good mixing induced by the strong surface flow may cause the phenomenon that there is almost no temperature fluctuation on droplet surface. When the droplet height decreases to less than $1 \mathrm{~mm}$, thermal waves appear and propagate along the circumferential or radial direction. We choose the surface infrared thermal imaging at $h=0.6 \mathrm{~mm}$ to illustrate the thermal pattern, which is characterized by the curved stripes traveling in the azimuthal direction, as shown in Fig. 5(b). This kind of thermal pattern is similar to the hydrothermal waves reported by Smith and Davis [26]. Furthermore, the similar pattern of the curved stripes in the sessile droplet evaporation process has also been observed by Sobac et al. [15] and Sefiane et al. [27]. They called this pattern as the hydrothermal waves. 

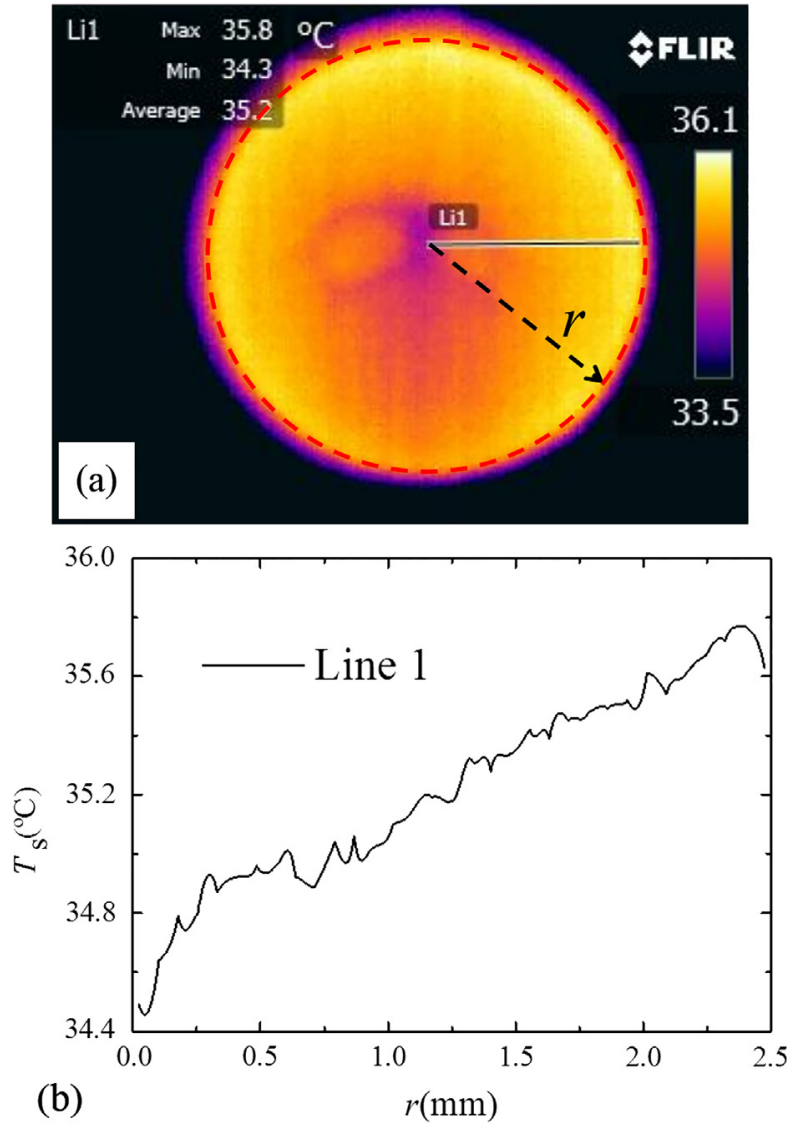

Fig. 3. The temperature distribution on the droplet surface. (a) The position of line 1 on infrared image. (b) Temperature distribution data obtained by line 1 .
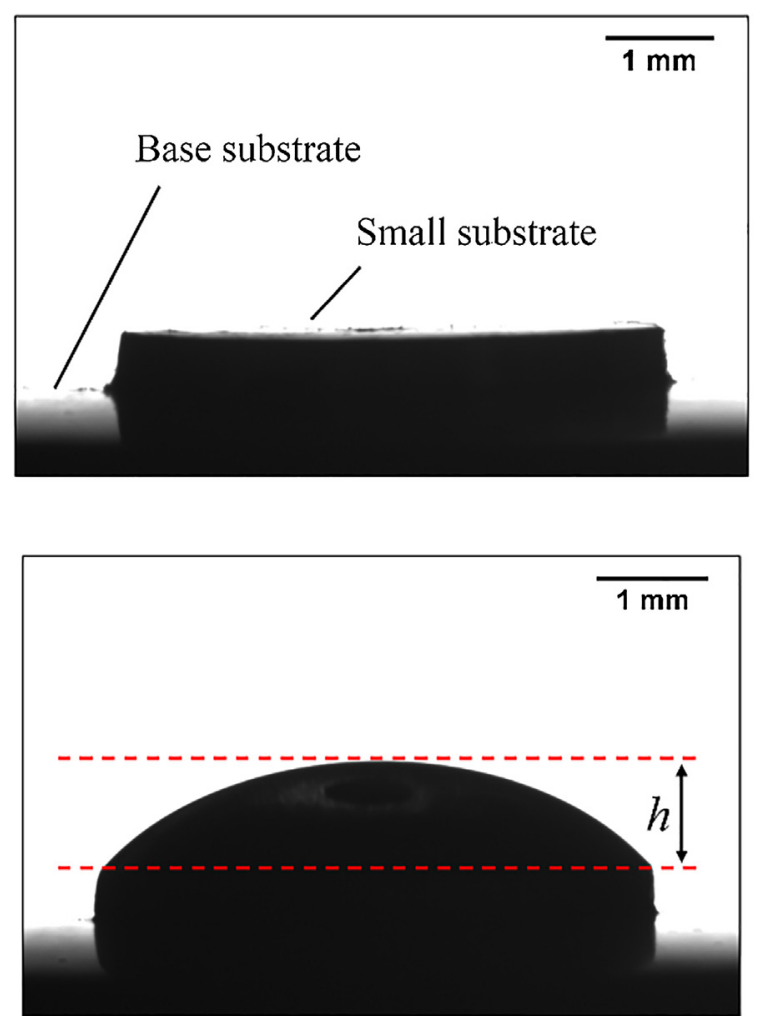

Fig. 4. The shape of small substrate and droplet.
Thus, the thermal pattern shown in Fig. 5(b) can be regarded as the hydrothermal waves. With the further decrease of the droplet height, the rosebud pattern is detected to generate near the triple line and shrink toward the center, as exhibited in Fig. 5(c) and 5(d).

In order to compare the thermal patterns of evaporation droplet on different substrates with different thermal properties, the droplet evaporation on an aluminum substrate has also been investigated. Thermal patterns of the isopropanol droplet evaporation on a copper or aluminum substrate are given in Fig. 6. When the droplet evaporates on copper substrate at $h=0.6 \mathrm{~mm}$, a forked pattern appears on the droplet surface at the low substrate temperature of $T_{\mathrm{w}}=30^{\circ} \mathrm{C}$, as shown in Fig. 6(a). Because the evaporation rate is relatively small, the temperature fluctuation is weak. When $T_{\mathrm{w}}=45^{\circ} \mathrm{C}$, the temperature fluctuation on the free surface appears as hydrothermal waves, as indicated in Fig. 6(b). Furthermore, when $T_{\mathrm{w}}$ is increased to $60^{\circ} \mathrm{C}$, the thermal pattern is indicated as straight spokes. Moreover, the wave number increases with the substrate temperature, which is consistent with the result of Brutin et al. [16]. According to the research of Wang et al. [28], the formation of new waves in the azimuthal direction can enhance the diffusion of momentum and thermal energy to the adjacent liquid. In present work, when the substrate temperature increases, the droplet can get more energy from the substrate to form new waves. Therefore, the wave number increases.

When the droplet height is fixed at $h=0.6 \mathrm{~mm}$, the thermal patterns induced by the droplet evaporation on an aluminum substrate are depicted in Fig. 6(d-f). As mentioned above, the small substrate is glued with the base substrate. During the experiment, the temperature distribution of base substrate is uniform. However, because the thermal conductivity of aluminum is less than that of copper, the temperature distributions of two small substrates are different when the base substrate temperature is the same. When the droplet evaporates on the aluminum substrate, the regular petal-like thermal pattern is recorded. The petal-like waves always emerge near the droplet edge, and then some of the waves will shrink to the droplet center during the evaporation process. At the same time, the area where the wave vanished will be occupied by the adjacent waves. It should be noticed that the total wave number was almost a constant. In addition, as shown in Fig. 7, the thermal pattern will fill the whole surface with the increase of the substrate temperature, which is similar to the result on copper substrate.

Fig. $6(\mathrm{~g}-\mathrm{i})$ exhibit the surface thermal patterns on aluminum substrate at $h=0.7 \mathrm{~mm}$. It is found that the surface thermal patterns are similar to those at $h=0.6 \mathrm{~mm}$, except at $T_{\mathrm{w}}=30^{\circ} \mathrm{C}$. Fig. $6(\mathrm{~g})$ shows that the thermal pattern looks like a forked pattern at $T_{\mathrm{w}}=30^{\circ} \mathrm{C}$. Because this pattern is different from other patterns at the same height, several experiments were carried out to confirm its existence. Compare Fig. 6(h, i) with Fig. 6(e, f) respectively, it can be found that the wave number increases with the increase of the droplet height. According to the report of Sefiane et al. [14], during the whole evaporation lifetime of sessile methanol and ethanol droplets, the total wave number will decrease with the decrease of the droplet height. They pointed out that the surface temperature gradient becomes small when the droplet height decreases. Therefore, the driving force for thermocapillary flow decreases, which will result in fewer thermal waves. However, when the droplet height is high enough or low enough, the thermal pattern of droplet evaporation on aluminum substrate is similar to that on copper substrate. For example, when $T_{\mathrm{w}}=50^{\circ} \mathrm{C}$, there is no thermal pattern at $h=1 \mathrm{~mm}$, but the rosebud pattern appears at $h=0.4 \mathrm{~mm}$, as shown in Fig. 7. This phenomenon is also similar to that on copper substrate.

The variation of wave number with substrate temperature is shown in Fig. 8. It can be found that the wave number gradually increases with the increase of the substrate temperature at each droplet height. In addition, because the thermal conductivity of copper is higher than that of aluminum, the temperature of the small copper substrate is always higher than that of the aluminum one when the base substrate temperature is the same. Consequently, the wave number of the thermal 

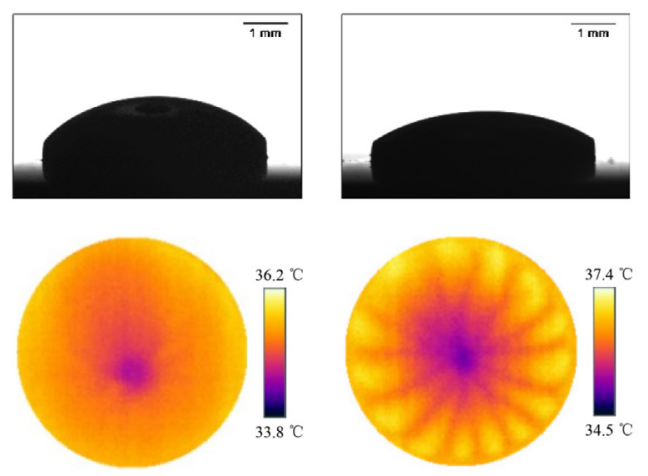

(a) $h=1 \mathrm{~mm}$

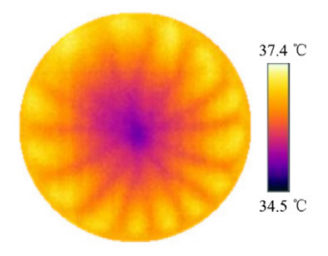

(b) $h=0.6 \mathrm{~mm}$
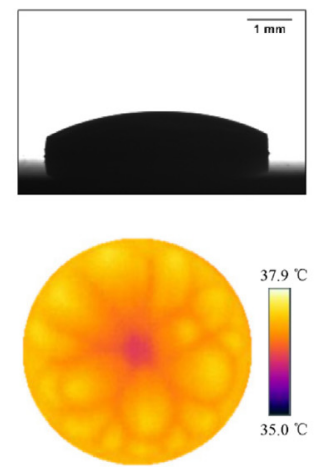

(c) $h=0.5 \mathrm{~mm}$
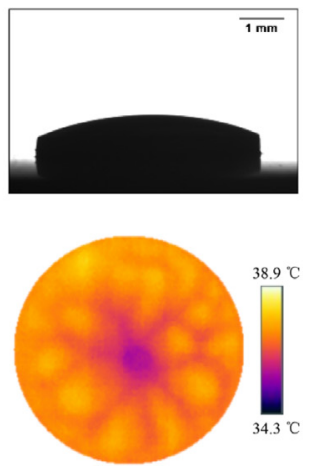

(d) $h=0.4 \mathrm{~mm}$

Fig. 5. Morphologies and thermal patterns of isopropanol droplet evaporating on copper substrate at $T_{\mathrm{w}}=40{ }^{\circ} \mathrm{C}$.

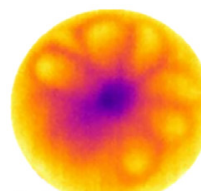

(a)

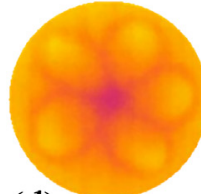

(d)

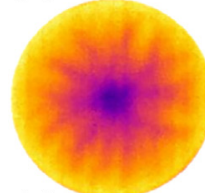

(g)

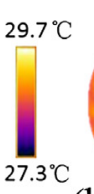

(b)

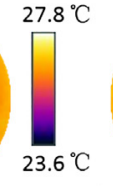

(e)

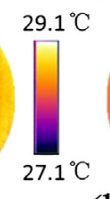

(h)

\section{)}
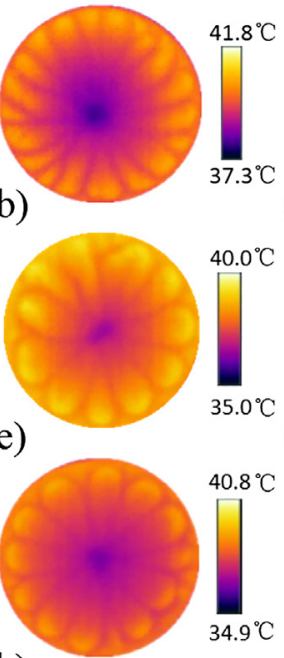

$T_{\mathrm{W}}=45^{\circ} \mathrm{C}$ (c)
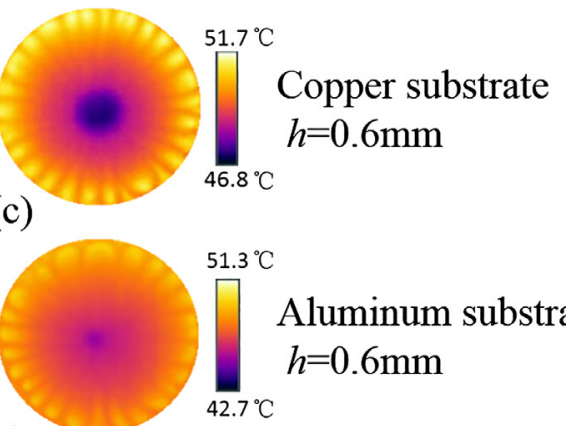

\section{Aluminum substrate} $h=0.6 \mathrm{~mm}$

(f)

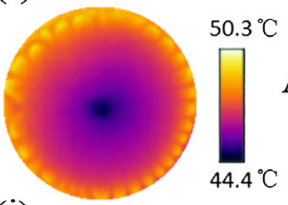

Aluminum substrate $h=0.7 \mathrm{~mm}$

$T_{\mathrm{w}}=30^{\circ} \mathrm{C}$

(i)

Fig. 6. Thermal patterns of isopropanol droplet evaporating on an aluminum or copper substrate.

pattern on copper is always larger than that on aluminum.

To compare the flow intensity of droplet on different substrates, the maximum temperature difference along the radial direction is analyzed. The infrared image at $T_{\mathrm{w}}=40^{\circ} \mathrm{C}$ and $h=0.7 \mathrm{~mm}$ was chosen as an example. Fig. 9 shows the maximum and average temperature difference along the radial direction on the line 1 (see Fig. 9(a)). It is worth mentioning that the temperature difference on copper substrate seems always higher than that on aluminum substrate. The average temperature difference on copper substrate is $2.00{ }^{\circ} \mathrm{C}$, which is larger than $1.68{ }^{\circ} \mathrm{C}$ on aluminum substrate. It hints that the temperature gradient along the droplet surface on copper substrate is larger than that on

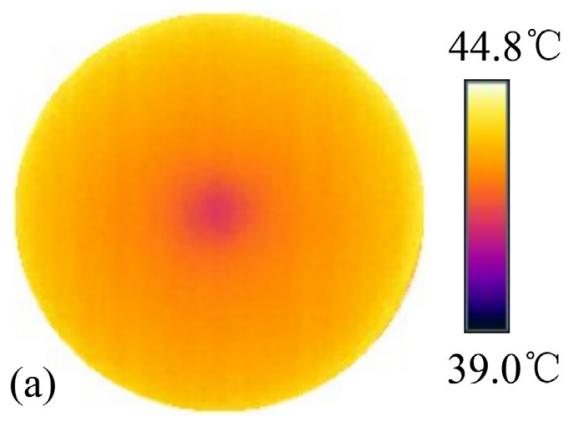

aluminum substrate. Therefore, the surface flow is more intense when the droplet evaporates on copper substrate.

\subsection{The relationship between evaporation rate and liquid flow}

During the experiment, the injection rate of syringe pump is adjusted to keep the liquid-vapor interface unmoving. Therefore, the global evaporation rate of the liquid droplet is equal to the injection rate of syringe pump. The evaporation rate increases with the substrate temperature, as exhibited in Fig. 10. It seems that the evaporation rate decreases a little with the droplet height. The main reason might be that

Fig. 7. Thermal pattern of droplet evaporate on aluminum substrate at $T_{\mathrm{w}}=50{ }^{\circ} \mathrm{C}$. (a) $h=1 \mathrm{~mm}$. (b) $h=0.4 \mathrm{~mm}$. 


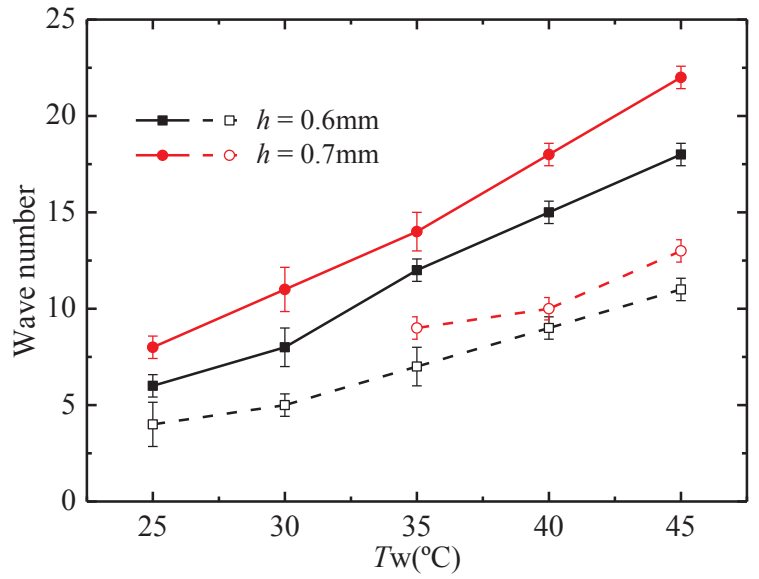

Fig. 8. Variation of wave number with substrate temperature and the droplet height. Solid lines: copper; Dotted lines: aluminum.
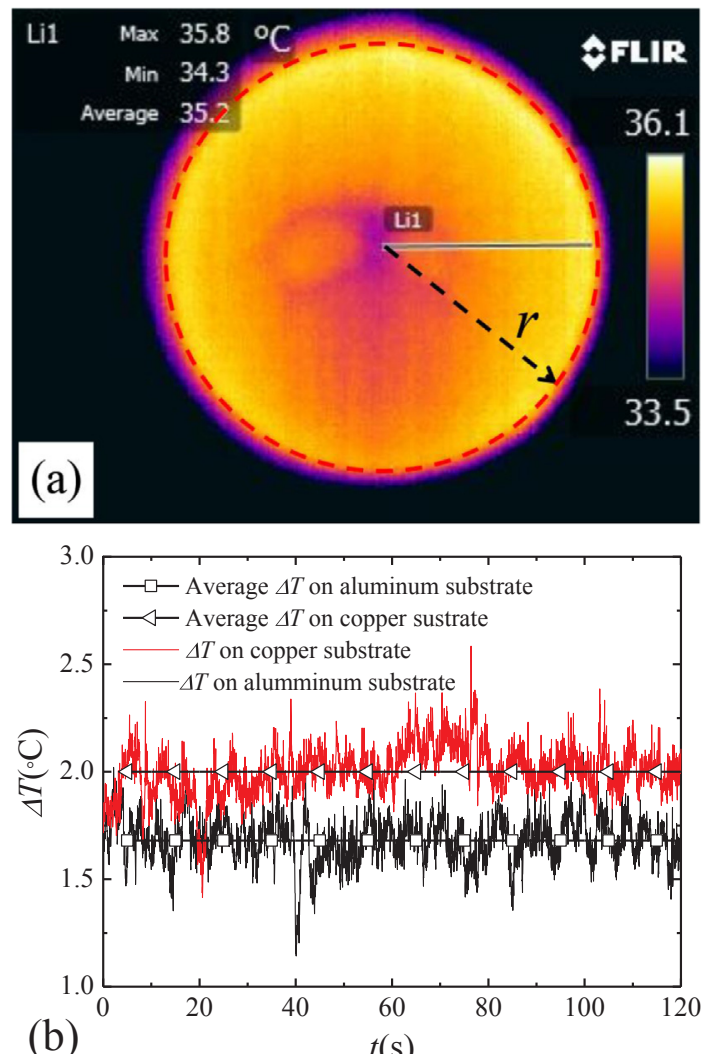

(b)

$t(\mathrm{~s})$

Fig. 9. The maximum and average temperature difference $(\Delta T)$ along radial direction of droplet surface on copper (red line) and aluminum substrate (black line) at $T_{\mathrm{w}}=40{ }^{\circ} \mathrm{C}$ and $\mathrm{h}=0.7 \mathrm{~mm}$. (a) The position of the line 1 on infrared image. (b) The maximum and average temperature difference on line 1.

the variation of the droplet surface area is very small when the droplet height varies from $0.6 \mathrm{~mm}$ to $0.8 \mathrm{~mm}$.

The droplet evaporation rate on copper substrate is always larger than that on aluminum substrate. It means that the evaporation process on copper substrate is stronger than that on aluminum substrate at the same droplet height and substrate temperature. Moreover, the surface temperature gradient of droplet on copper substrate is larger than that on aluminum substrate, the example is shown in Fig. 9. It means that when the evaporation process becomes strong, the radial temperature difference induced by evaporation becomes large. Therefore, the flow driven by the droplet evaporation on copper substrate is more intense.

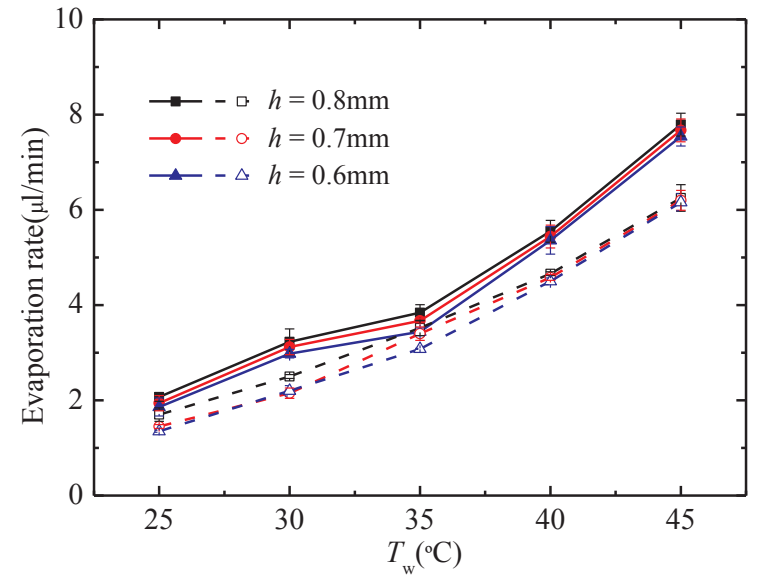

Fig. 10. Variation of evaporation rate with substrate temperature and the droplet height. Solid lines: copper; Dotted lines: aluminum.

Moreover, according to the research of Duan et al. [29], when the evaporation rate is high enough, thermal conduction cannot provide sufficient energy to maintain the droplet evaporation. Therefore, there must exist thermal convection when the evaporation rate is high enough. In this case, if the flow becomes more intense, the evaporation rate will increase, which means that the evaporation process and the flow intensity are coupled with each other. Flow enhancement can promote evaporation, which in turn enhance itself. Eventually, evaporation and liquid flow interact to achieve a new state. The new state should be higher evaporation rate and stronger flow for the case of a higher substrate temperature. At the same base substrate temperature, the surface temperature of copper substrate is higher than that of aluminum substrate. Consequently, when the droplet evaporates on copper substrate, the evaporation rate is higher and the liquid flow is more intense. Additionally, because the evaporation process and the liquid flow are coupled with each other, thermal patterns become more complex when evaporation rate increases. For example, comparing the thermal patterns showed in Fig. 6(b) and Fig. 6(d). It can be found that the wave number increases and the thermal pattern area of covering droplet surface is wider when the evaporation rate becomes higher.

\section{Conclusions}

Evolution of thermal patterns during steady state evaporation of sessile droplets was experimentally investigated by utilizing the highspeed infrared thermal imager and CMOS camera. Results show that the steady state evaporation process of isopropanol droplet induces the formation of heat convection. The main conclusions are summarized as follows.

(1) Thermal patterns depend on the droplet height. At a high droplet height, the surface temperature distribution is uniform and there is no thermal pattern at the droplet surface. Varieties of thermal patterns are observed with the decreasing of droplet height.

(2) Benefiting from the steady state evaporation of isopropanol droplets, the wave number of thermal pattern is recorded accurately. Actually, the wave number is proportional to the substrate temperature. In addition, compared with thermal patterns of droplets evaporate on aluminum substrate, it can be found that the wave number is always larger when droplets evaporate on copper substrate.

(3) Because the thermal properties of aluminum and copper are different, the thermal pattern is not the same when droplet evaporates on different substrate. When the droplet evaporates on copper substrate, forked pattern, curved hydrothermal waves and rosebud pattern are recorded. Meanwhile, on aluminum substrate, forked 
pattern, petal-like pattern and rosebud pattern are observed.

(4) The global evaporation rate and flow intensity are also influenced by the thermal properties of the substrate. In summary, the evaporation rate is higher and the surface flow is more intense when the droplet evaporates on copper substrate.

\section{Conflicts of interest}

None.

\section{Acknowledgement}

This work is supported by the National Natural Science Foundation of China (Grant No. 11532015) and the Fundamental Research Funds for the Central Universities (No. 2018CDXYDL0001)

\section{References}

[1] W.W. Deng, A. Gomez, Electrospray cooling for microelectronics, Int. J. Heat Mass Transf. 54 (2011) 2270-2275.

[2] J. Kim, Spray cooling heat transfer: the state of the art, Int. J. Heat Fluid Flow 28 (2007) 753-767.

[3] P. Calvert, Inkjet printing for materials and devices, Chem. Mat. 13 (2001) 3299-3305.

[4] Y.K. Kim, H.K. Na, S. Ham, D.H. Min, Mediating ordered assembly of gold nanorods by controlling droplet evaporation modes for surface enhanced Raman scattering, RSC Adv. 4 (2014) 50091-50096.

[5] D. Brutin, B. Scbac, B. Loquet, J. Sampol, Pattern formation in drying drops of blood, J. Fluid Mech. 667 (2010) 85-95.

[6] R.D. Deegan, O. Bakajin, T.F. Dupont, G. Huber, S.R. Nagel, T.A. Witten, Capillary flow as the cause of ring stains from dried liquid drops, Nature 389 (1997) 827-829.

[7] R.G. Picknett, R. Bexon, Evaporation of sessile or pendant drops in still air, J. Colloid Interf. Sci. 61 (1977) 336-350.

[8] K.S. Birdi, D.T. Vu, Wettability and the evaporation rates of fluids from solid surfaces, J. Adhes. Sci. Technol. 7 (1993) 485-493.

[9] M.E.R. Shanahan, C. Bourgès, Effects of evaporation on contact angles on polymer surfaces, Int. J. Adhes. Adhes. 14 (1994) 201-205.

[10] H. Hu, R.G. Larson, Evaporation of a sessile droplet on a substrate, J. Phys. Chem. B 106 (2002) 1334-1344.

[11] K. Gleason, H. Voota, S.A. Putnam, Steady-state droplet evaporation: contact angle influence on the evaporation efficiency, Int. J. Heat Mass Transf. 101 (2016) 418-426.

[12] H. Hu, R.G. Larson, Marangoni effect reverses coffee-ring depositions, J. Phys. Chem. B 110 (2006) 7090-7094.

[13] W.D. Ristenpart, P.G. Kim, C. Domingues, J. Wan, H.A. Stone, Influence of substrate conductivity on circulation reversal in evaporating drops, Phys. Rev. Lett. 99 (2007) 234502.

[14] K. Sefiane, J.R. Moffat, O.K. Matar, R.V. Craster, Self-excited hydrothermal waves in evaporating sessile drops, Appl. Phys. Lett. 93 (2008) 074103.

[15] B. Sobac, D. Brutin, Thermocapillary instabilities in an evaporating drop deposited onto a heated substrate, Phys. Fluids 24 (2012) 032103.

[16] D. Brutin, B. Sobac, F. Rigollet, C.L. Niliot, Infrared visualization of thermal motion inside a sessile drop deposited onto a heated surface, Exp. Therm. Fluid Sci. 35 (2011) 521-530.

[17] K. Sefiane, Y. Fukatani, Y. Takata, J. Kim, Thermal patterns and hydrothermal waves (HTWs) in volatile drops, Langmuir 29 (2013) 9750-9760.

[18] C. Bouchenna, M.A. Saada, S. Chikh, L. Tadrist, Generalized formulation for evaporation rate and flow pattern prediction inside an evaporating pinned sessile drop, Int. J. Heat Mass Transf. 109 (2017) 482-500.

[19] M.A. Mahmud, B.D. Macdonald, Experimental investigation of interfacial energy transport in an evaporating sessile droplet for evaporative cooling applications, Phys. Rev. E 95 (2017) 012609.

[20] M. Pack, H. Hu, D.O. Kim, X. Yang, Y. Sun, Colloidal drop deposition on porous substrates: competition among particle motionevaporation, and infiltration, Langmuir 31 (2015) 7953-7961.

[21] A. Daiacute, M. Bouaziz, J. Bresson, M. Grisenti, Surface temperature of hydrocarbon droplet in evaporation, J. Thermophys. Heat Transf. 13 (2012) 553-555.

[22] F. Carle, B. Sobac, D. Brutin, Hydrothermal waves on ethanol droplets evaporating under terrestrial and reduced gravity levels, J. Fluid Mech. 712 (2012) 614-623.

[23] P.J. Sáenz, K. Sefiane, J. Kim, O.K. Matar, P. Valluri, Evaporation of sessile drops: a three-dimensional approach, J. Fluid Mech. 772 (2015) 705-739.

[24] Y. Fukatani, D. Orejon, Y. Kita, Y. Takata, J. Kim, K. Sefiane, Effect of ambient temperature and relative humidity on interfacial temperature during early stages of drop evaporation, Phys. Rev. E 93 (2016) 043103.

[25] G.W. Gokel, J.A. Dean, Dean's Handbook of Organic Chemistry, McGraw-Hill handbooks, 2004.

[26] M.K. Smith, S.H. Davis, Instabilities of dynamic thermocapillary liquid layers. Part 1. Convective instabilities, J. Fluid Mech. 132 (1983) 119-144.

[27] K. Sefiane, A. Steinchen, R. Moffat, On hydrothermal waves observed during evaporation of sessile droplets, Colloid Surf. A-Physicochem. Eng. Asp. 365 (2010) 95-108.

[28] B.F. Wang, Z.W. Guo, D.J. Ma, D.J. Sun, Instabilities and pattern evolution in a vertically heated annulus, Sci. China-Phys. Mech. Astron. 56 (2013) 257-262.

[29] F. Duan, C.A. Ward, Surface excess properties from energy transport measurements during water evaporation, Phys. Rev. E 72 (2005) 056302. 